\title{
APLICAÇÃO DE SISTEMA ESPECIALISTA PARA CONTROLE DA FLOTAÇÃO CONVENCIONAL DO CONCENTRADOR 2 DA SAMARCO*
}

\author{
André Nogueira ${ }^{1}$ \\ Fernando Andrade Pires ${ }^{2}$ \\ Flávio Thimótio da Silva ${ }^{3}$ \\ Marcelo Montalvão Gontijo ${ }^{4}$
}

\section{Resumo}

A etapa de flotação convencional do Concentrador 2 ocorre em um circuito com 13 tanques de flotação que recebem o produto dos ciclones limpadores $(-0,15 \mathrm{~mm}$ $+0,030 \mathrm{~mm}$ ). O concentrado desta etapa contribui com aproximadamente $80 \%$ do volume de produção do concentrador. A necessidade da implantação do sistema de controle avançado de processos (SCAP) advém da alta heterogeneidade na alimentação do concentrador, uma vez que a Samarco não conta com pilha de homogeneização. O objetivo principal do projeto foi maximizar a recuperação metálica da flotação convencional e reduzir a variabilidade do teor de sílica no concentrado final. Aliado ao sistema de análise de imagem de espuma e ao analisador de sílica online, a ferramenta demostrou que, além de atingir os objetivos esperados, trouxe estabilização ao processo e padronização da operação.

Palavras-chave: Flotação; Sistema especialista; Análise de imagem.

\section{APPLICATION OF EXPERT SYSTEM FOR CONTROL OF THE CONVENTIONAL FLOTATION OF CONCENTRATOR 2 OF SAMARCO}

\section{Abstract}

The conventional flotation process in Concentrator 2 occurs in a circuit with 13 flotation tanks which receives the product from the cyclone cleaners $(-0,15 \mathrm{~mm}$ $+0,030 \mathrm{~mm}$ ). The concentrate of this stage contributes with $80 \%$ of the production volume of the concentrator. The necessity of the implementation of an advanced process control system (SCAP) arises from the high heterogeneity of the feed, since Samarco does not have a homogenization stack. The main goal of the project was to maximize iron recovery of conventional flotation and reduce the variability of the silica content in the final concentrate. Coupled with the froth image analysis and the online silica analyzer system, the tool demonstrated that in addition to achieving the expected objectives, brought stabilization and standardization to the process operation.

Keywords: Flotation; Expert system; Image analysis.

Engenheiro de Processo, Samarco Mineração S/A. Mariana, MG, Brasil.

Engenheiro de Automação, Samarco Mineração S/A. Mariana, MG, Brasil.

Chefe de Departamento de Engenharia de Processos, Samarco Mineração S/A. Mariana, MG, Brasil.

4 Gerente de Controle Avançado de Processos, Metso Brasil. Belo Horizonte, MG, Brasil. 


\section{INTRODUÇÃO}

A Samarco utiliza ferramentas de controle avançado com ganho comprovado desde o ano de 2007. A empresa entende que a utilização de tecnologias como essa tornou-se uma necessidade constante.

O sistema especialista suplementa os Controladores Lógicos Programáveis (CLP) e os Sistemas de Controle Distribuídos (DCS) e continuamente maximizam a rentabilidade da operação da planta. Estes sistemas automaticamente recebem informações do PLC ou DCS, e usam várias técnicas avançadas para elaborar novos set-points apropriados para o processo com uma frequência muito alta. Estes set-points são estabelecidos para continuamente perseguir os objetivos da estratégia de controle. $\mathrm{O}$ método de controle que melhor traduz esta estratégia e apresenta os melhores resultados em flotação é a lógica fuzzy, que consiste em um conjunto de regras que reproduzem as melhores práticas operacionais. Aliado ao sistema especialista está o sistema de análise de imagem de espuma, responsável por medir a velocidade de arraste, fundamental para o controle. Outra tecnologia consolidada e patenteada pela Samarco é o analisador de sílica online, que fornece informações sobre a tendência da sílica no concentrado, bem como os analisadores de RX, que fornecem a tendência do ferro no rejeito.

Estas ferramentas contribuíram para maximizar a recuperação metálica da flotação convencional e reduzir a variabilidade do teor de sílica no concentrado final.

\section{MATERIAIS E MÉTODOS}

O sistema especialista foi implantado na etapa de flotação convencional do Concentrador 2, que consiste em 13 máquinas de flotação tipo Tank Cell, cuja alimentação é proveniente do underflow dos ciclones limpadores, com diâmetro de 15 polegadas, cuja distribuição granulométrica encontra-se na faixa de $-0,15 \mathrm{~mm}$ até $+0,030 \mathrm{~mm}$.

O circuito é composto por 3 unidades rougher, 3 unidades cleaner, 2 unidades recleaner, 2 unidades scavenger da rougher, 2 unidades scavenger da cleaner e por 1 unidade cleaner da scavenger (Figura 1).

Para medição da velocidade de deslocamento da espuma foram instaladas 8 câmeras distribuídas nas etapas rougher, cleaner, recleaner e scavengers. 


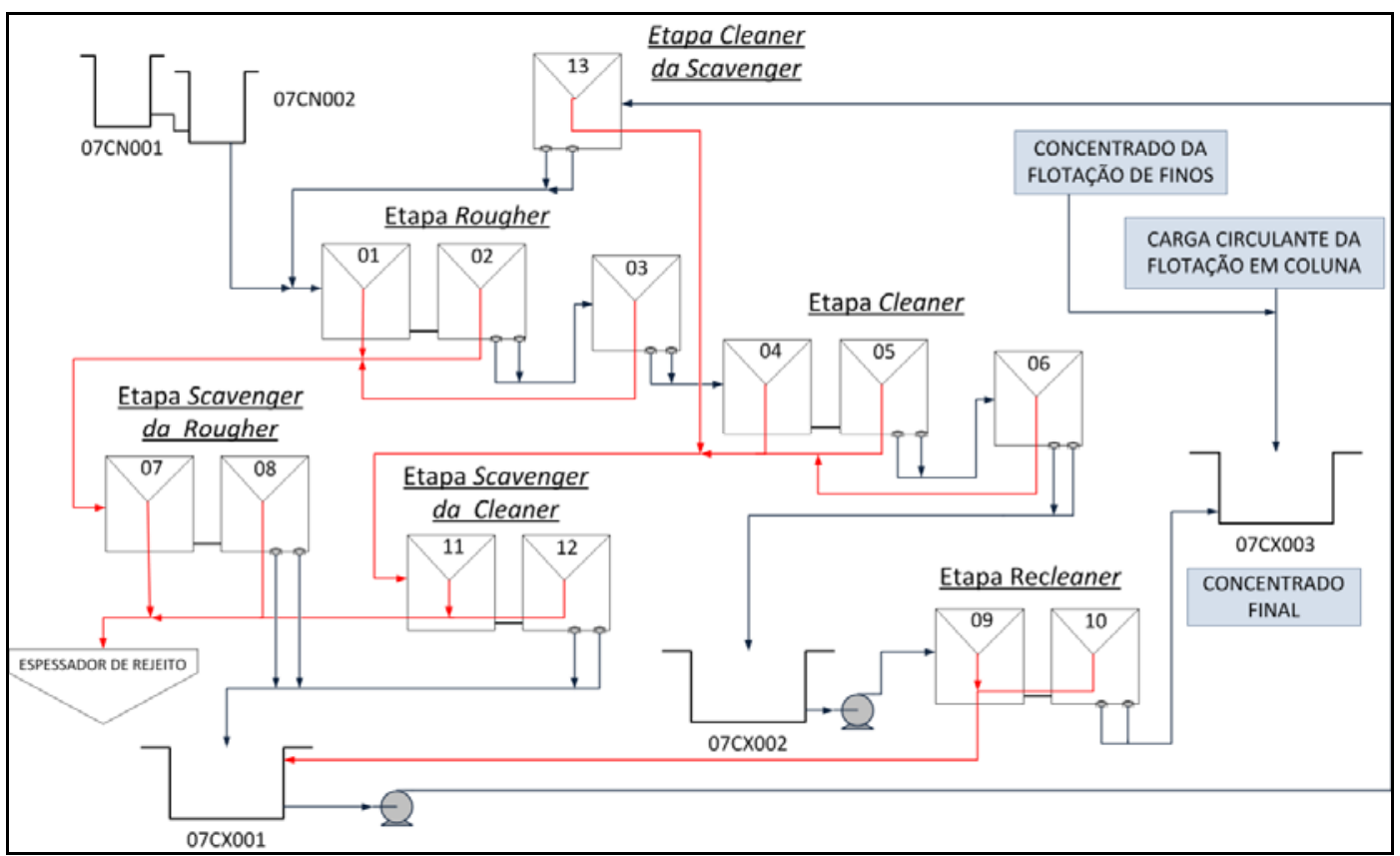

Figura 1. Circuito de flotação convencional do Concentrador 2.

\subsection{Definição das Variáveis Controladas e Manipuladas}

A primeira etapa para a implementação do sistema envolve a definição das variáveis controladas e manipuladas. A Variável Controlada é a grandeza ou condição que é medida e controlada(Ex.:\%Silica no Concentrado). A Variável Manipulada é a grandeza ou a condição modificada pelo controlador, de modo que o valor da variável controlada atinja o set-point desejado(Ex.:Nível da Camada de Espuma de Flotação). As relações entre todas as variáveis são definidas pela estratégia de controle, que são construídas com base no conhecimento dos operadores mais experientes, dos engenheiros de processo da planta e dos engenheiros responsáveis pela configuração do sistema.

$\mathrm{Na}$ prática diária, operadores experientes dos circuitos de flotação utilizam as características denominadas "aspecto" visuais da espuma para a tomada de decisões e parametrização do processo. Dentre todas as propriedades visuais da espuma, a que se mostra mais adequada para a predição do teor de sílica no concentrado, é o deslocamento no sentido do transbordo [1].

O controle de velocidade da espuma foi adotado de forma que o sistema manipula o nível de cada tanque para garantir a estabilidade da taxa de flotado.

Outra variável fundamental para construção da estratégia de controle foi a tendência do comportamento da sílica no concentrado da flotação convencional, fornecida pelo analisador online desenvolvido pela Samarco. Trata-se de um conjunto de mecanismo, câmera de vídeo, microcomputador e software aplicados à preparação e análises de amostras num ciclo de resultados em torno de 17 minutos [2]. Deste modo, o set-point de velocidade em cada tanque é definido a cada novo resultado do analisador de sílica online, bem como os set-points de dosagem de amina e amido.

\subsection{Definição do Objetivo de Sílica no Concentrado da Flotação}

Além dos resultados do analisador online, a definição do objetivo de sílica no concentrado da flotação convencional também leva em conta o teor de sílica da alimentação do espessador de concentrado, proveniente do laboratório, e do 
objetivo de sílica no concentrado final informado pelo operador, que depende da especificação do produto a ser beneficiado. (Equação 1).

$$
\text { ObjetivoSilicaConcConv }=\frac{\text { ObjetivoSilicaEspessador }}{1-\left(\frac{\text { SilicaConcConvAtual }- \text { SilicaEspessadorAtual }}{\text { SilicaConcConvAtual }}\right)}
$$

\subsection{Definição da Lógica de Controle Avançado}

Após a determinação do objetivo de sílica no concentrado convencional e da relação entre as variáveis manipuladas e controladas definiu-se que o método de controle a ser utilizado seria baseado em lógica fuzzy, técnica essa com resultados comprovados em aplicações para flotação. O objetivo principal do método consiste em mimetizar o conhecimento dos operadores sobre o processo, incorporando as estratégias de controle ao sistema. A implementação da lógica Fuzzy permite que estados indeterminados possam ser tratados por dispositivos de controle. Desse modo, é possível avaliar conceitos linguisticos não quantificáveis, por exemplo avaliar os Teores de Sílica no Concentrado e Ferro no Rejeito (Alto, Estável, Baixo, etc.), (Figura 2).

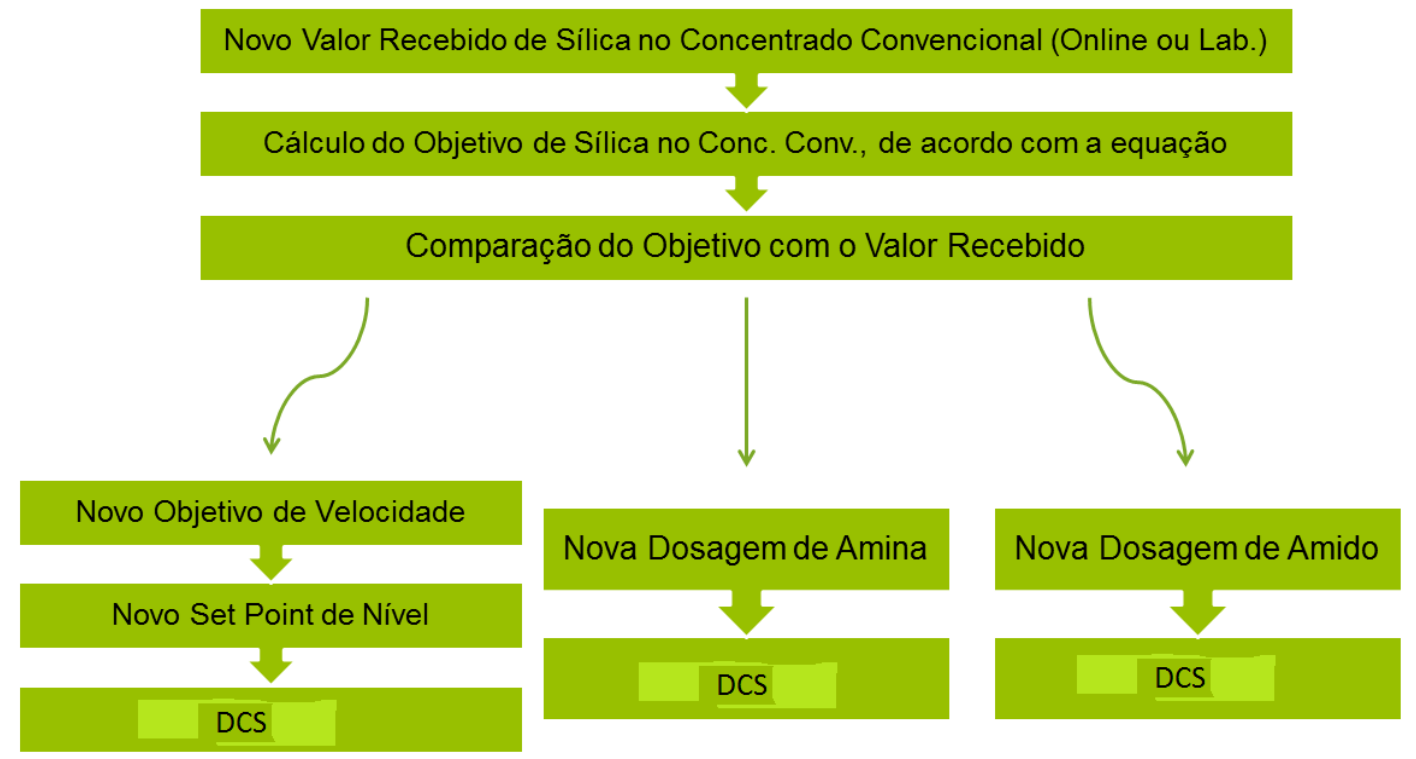

Figura 2. Visão geral da estratégia de controle do SCAP

Em resumo, identificadas as entradas do sistema, foram traçados os conjuntos lingüísticos Fuzzy para cada uma delas e estabelecidas às relações em regras do tipo "If...than". De posse das ações definidas para cada situação, foi possível Desfuzzyficar as variáveis e como resultado tem-se as ações de controle otimizada. A última etapa envolveu a consolidação das regras de controle e inclusão de melhorias identificadas durante a operação assistida do sistema.

\section{RESULTADOS E DISCUSSÃO}

Foi realizado um teste preliminar durante 5 dias, com acompanhamento da equipe de implantação do sistema especialista. Um banco de dados foi levantado, 
considerando a utilização horária do sistema e os dados de laboratório, como \%Fe no rejeito e \%SiO2 no concentrado. As informações sobre dosagem de reagentes também foram levantadas. Os índices avaliados foram:

- Média e desvio padrão do \%SiO2 do concentrado da flotação (resultado de laboratório).

- Média e desvio padrão do \%SiO2 no concentrado final.

- Média e desvio padrão de \%Fe no rejeito da flotação convencional.

- Média da dosagem de amido.

- Média da dosagem de amina.

Os ganhos são calculados sempre com relação ao desempenho do sistema especialista desligado (Equação 2):

$$
G \quad \%=\left(\frac{\text { Média }_{\text {ON }}-\text { Média }_{\text {OFF }}}{\text { Média }}\right) \cdot 100
$$

Os principais ganhos obtidos nos testes preliminares foram a melhoria na qualidade do concentrado final de $4 \%$ e redução da média geral de ferro no rejeito de $12 \%$ além de uma pequena diminuição do consumo de reagentes (Tabela 1). Houve também redução da variabilidade de sílica no concentrado da flotação de $15 \%$ e em $40 \%$ no concentrado final. Ainda na etapa de flotação convencional, a variabilidade de ferro no rejeito foi reduzida em $35 \%$ (Tabela 2 ).

Tabela 1. Resultados do teste preliminar de desempenho

\begin{tabular}{cc}
\hline Índice & Ganho \\
\hline$\%$ SiO2 concentrado final & $-4,2 \%$ \\
\hline \%SiO2 do concentrado flotação & $-2,9 \%$ \\
\hline \%Fe no rejeito & $-12,6 \%$ \\
\hline Dosagem de Amido & $-1,2 \%$ \\
\hline Dosagem de Amina & $-1,2 \%$ \\
\hline
\end{tabular}

Tabela 2. Resultados de variabilidade das variáveis de processo do teste de desempenho

\begin{tabular}{cc}
\hline Índice & Ganho \\
\hline Desvio Padrão da sílica (concentrado flotação) & $-15,1 \%$ \\
\hline Desvio Padrão do percentual de ferro no rejeito & $-35,3 \%$ \\
\hline Desvio Padrão da sílica (concentrado final) & $-40,2 \%$ \\
\hline
\end{tabular}

O teste final de desempenho do sistema está programado para ocorrer em Maio, devido a necessidade de fazer um treinamento mais específico para os operadores, técnicos e chefes de equipe visando sanar todas as dúvidas existentes e corretas parametrizações do sistema.

Para assegurar estes resultados o SCAP necessita estar sempre traduzindo a realidade de processo. Estudos especializados mostram que projetos implementados com sucesso e metas atendidas, perdem $50 \%$ da eficiência apenas alguns meses após a implementação, caso não haja um plano de manutenção prévio das regras implementadas para controle.

As alterações que podem deteriorar a eficiência de um sistema especialista ao longo do tempo incluem: mudanças no fluxograma de processo, definição de um novo 
objetivo, mudanças na instrumentação ou outros tipos de melhoria no DCS podem se tornar fatores que alteram o desempenho do SCAP.

Finalmente, para evitarem-se perdas de eficiência do sistema ao longo do tempo, recomenda-se a criação de uma estrutura de manutenção (tanto do sistema quanto dos analisadores online).

\section{CONCLUSÃO}

O controle de processo através do sistema especialista aliado às informações provenientes dos analisadores online contribuiu para aumentar a recuperação metálica da flotação convencional do Concentrador 2 , evidenciada pela queda do teor de ferro no rejeito. Além disso, observou-se uma redução na variabilidade do processo obtida através da padronização do controle.

Para continuidade dos ganhos obtidos foi criada uma estrutura de manutenção do sistema especialista e dos analisadores online, fundamentais para o bom desempenho do sistema.

O sistema especialista será implantado nas outras operações unitárias do concentrador 2, bem como, nos demais concentradores.

\section{Agradecimentos}

Os autores agradecem aos operadores e técnicos de produção do Concentrador 2 em especial ao Especialista de Processo Paulo Mapa pela importante contribuição no projeto. Ao Técnico de Processo Leonidas Angelo Junior pelo apoio na manutenção dos analisadores online. Ao Engenheiro de Processo Vitor Hugo. A toda equipe de operação, engenheiros e gestores pelo apoio durante e após a implementação do sistema especialista na flotação da Usina 2 de Germano.

\section{REFERÊNCIAS}

1 Silva FT. Propriedades visuais de espuma para modelagem dos teores da flotação convencional da Samarco. Belo Horizonte. Dissertação [Mestrado em Tecnologia Mineral] - UFMG; 2011.

2 Silva AJ. Sistema de Medição de Teor de Sílica em Minério de Ferro por Análise de Imagem. Tecnologia em Metalurgia e Materiais. 2005;1(3):64-67. 